\title{
Functional Recovery Following the Transplantation of Olfactory Ensheathing Cells in Rat Spinal Cord Injury Model
}

\author{
Durai Murugan Muniswami, George Tharion \\ Department of Physical Medicine and Rehabilitation, Christian Medical College, Vellore, India
}

Study Design: Olfactory ensheathing cells (OECs) from rat olfactory mucosa were cultured, characterized, and transplanted into a rat model of spinal cord injury (SCI).

Purpose: To evaluate different doses of OECs in a rat model of SCl.

Overview of Literature: SCI causes permanent functional deficit because the central nervous system lacks the ability to perform spontaneous repair. Cell therapy strategies are being explored globally. The clinical use of human embryonic stem cell is hampered by ethical controversies. Alternatively, OECs are a promising cell source for neurotransplantation. This study aimed to evaluate the efficacy of different doses of allogenic OEC transplantation in a rat model of SCl.

Methods: OECs were cultured from the olfactory mucosa of Albino Wistar rats; these cells were characterized using immunohistochemistry and flow cytometry. Rats were divided into five groups ( $n=6$ rats each). In each group, different dosage $\left(2 \times 10^{5}, 5 \times 10^{5}\right.$, $10 \times 10^{5}$, and $>10 \times 10^{5}$ ) of cultured cells were transplanted into experimentally injured spinal cords of rat models. However, in the SCI group, only DMEM (Dulbecco's modified Eagle's medium) was injected. Rats were followed up upto 8 weeks post-transplantation. The outcome of transplantation was assessed using the Basso, Beattie, Bresnahan (BBB) scale; motor-evoked potential studies; and histological examination.

Results: Cultured cells expressed $41 \%$ of p75NTR, a marker for OEC, and 35\% of anti-fibronectin, a marker for olfactory nerve fibroblast. These cells also expressed $\mathrm{S} 100 \beta$ and glial fibrillary acid protein of approximately $75 \%$ and $83 \%$, respectively. All the transplanted groups showed promising BBB scores for hind-limb motor recovery compared with the SCl group $(p<0.05)$. A motor-evoked potential study showed increased amplitude in all the treated groups compared with the SCI. Green fluorescent protein-labeled cells survived in the injured cord, suggesting their role in the transplantation-mediated repair. Transplantation of $5 \times 10^{5}$ cells showed the best motor outcomes among all the doses.

Conclusions: OECs demonstrated a therapeutic effect in rat models with the potential for future clinical applications.

Keywords: Basso, Beattie, Bresnahan score; Drop-weight injury; Electromyographic amplitude; Histology; Olfactory ensheathing cell transplantation

Received Feb 3, 2018; Revised Mar 6, 2018; Accepted Apr 17, 2018

Corresponding author: Durai Murugan Muniswami

Department of Physical Medicine and Rehabilitation, Christian Medical College, Vellore-632004, Tamil Nadu, India

Tel: +91-0416-2282158, Fax: +91-0416-2232035, E-mail: durai.morgan@gmail.com 


\section{Introduction}

Traumatic spinal cord injury causes hemorrhage, edema, ischemia, and hypoxia that result in tissue damage and cell death. This primary injury is followed by secondary cascades of inflammation, free radical creation, and excitotoxic damage that cause the death of neurons and myelinating oligodendrocytes. Astrocytes proliferate in the injured area and form a glial scar. This loss of neurons, axons, and demyelination results in permanent paralysis after spinal cord injury [1]. An injured spinal cord lacks the ability to regenerate spontaneously; therefore, there is a need for exogenous strategies for the recovery of functions. Cellbased strategies using embryonic stem cells, neural stem cells, mesenchymal stem cells, Schwann cells, and olfactory ensheathing cells (OECs) to regenerate neural tissue are being explored in various centers worldwide $[2,3]$.

Neurogenesis occurs in the olfactory system throughout the adult life of mammals. Olfactory neurons are constantly replaced; this process is facilitated by OECs that are specialized glial cells [4]. The OEC possess unique properties of both the central nervous system (CNS) glial cell astrocytes and the peripheral nervous system glial Schwann cells. OEC can reside in the CNS environment like astrocytes and facilitate axonal regrowth as Schwann cells $[5,6]$. OEC secrete brain-derived neurotrophic factor, neuregulins, nerve growth factor [7], glial derived neurotrophic factor [8], NT-3, and NT-4. Numerous studies have demonstrated the ability of OECs to remyelinate axons in in-vivo models [9].These exceptional qualities of OEC have made researchers consider these cells for nervous system repair.

In the last decade, animal studies have shown that transplantation of cultured OEC and olfactory nerve fibroblasts (ONF) from the olfactory bulb elicits functional reconnection of the severed axons and regeneration in spinal cord injuries [10,11]. Barbour et al. [11] demonstrated a remarkable increase in neuronal cell survival following OEC transplantation in the rat model. In addition to promoting neurite growth, subsequent research reveals guidance, angiogenic, neuroprotective, immune modulation, and remyelination properties as the underlying mechanisms [12]. OEC transplantation restored nerve conduction [13] and aided locomotor recovery following SCI [14].

However, the extent to which OEC transplants aid functional recovery remains unclear. Several international studies have reported varying outcomes of OEC transplantation in SCI. This study aimed to evaluate the effect of rat olfactory mucosa OEC in a SCI rat model and determine the optimum dose for maximum therapeutic effect. In this study, OECs were cultured, characterized, and transplanted with different dosages $\left(2 \times 10^{5}, 5 \times 10^{5}, 10 \times 10^{5}\right.$, and $\left.>10 \times 10^{5}\right)$ in a rat model of SCI. The treatment outcomes were measured using the motor score (Basso, Beattie, Bresnahan [BBB] score), electrophysiological studies (electromyographic [EMG]), and histological examination.

\section{Materials and Methods}

Adult Albino Wistar rats were used for the study; they were obtained from the animal house of the institution (Christian Medical College, Vellore, Tamil Nadu). The study was approved by the institutional review board and the institutional animal ethical committee of Christian Medical College, Vellore (IAEC approval no., 1/2010).

\section{Collection of rat olfactory mucosa}

Olfactory mucosa of male Wistar rats was collected. The animals were anesthetized with intraperitoneal injection of ketamine and xylazine. The olfactory mucosa was removed from the posterior region of the nasal septum, was dissected, and immediately placed in Dulbecco's modified Eagle's medium/Hams F12 (DMEM/F12; Gibco-Invitrogen, Carlsbad, CA, USA). The olfactory mucosa was readily identified owing to its yellowish color, striation of the lamina propria, and its posterior position on the nasal septum. The researchers were careful to avoid the anterior edge of the olfactory mucosa that could be contaminated with respiratory epithelium. The excised olfactory mucosas were placed in an ice-cold culture medium mixture (1:1, v:v) of Dulbecco's modified Eagle medium and Ham's F12 (DMEM/F12, Gibco-Invitrogen) supplemented with $100 \mathrm{U} / \mathrm{mL}$ penicillin, $100 \mu \mathrm{g} / \mathrm{mL}$ streptomycin, and 25 $\mathrm{ng} / \mathrm{mL}$ amphotericin-B; thereafter, they were transported to the cell culture laboratory within 30 minutes and processed for cell culture.

\section{Culture of olfactory ensheathing cells from the lami- na propria}

The olfactory mucosa was washed twice in Hank's bal- 
anced salt solution (HBSS) and incubated for $30 \mathrm{~min}$ utes at $37^{\circ} \mathrm{C}$ in $1 \mathrm{~mL}$ of $2.4 \mathrm{U} / \mathrm{mL}$ Dispase II (Bacillus polymyxa; Roche Diagnostics, Mannheim, Germany). Digestion was halted by adding HBSS, calcium- and magnesium-free (Gibco-Invitrogen). The olfactory epithelium was carefully peeled away from the lamina propria using a microspatula under a dissection microscope. The lamina propria had a brownish appearance, while the epithelium was whitish. The lamina propria was washed in HBSS and finely chopped into pieces using a scalpel blade. Thereafter, the tissue was incubated at $37^{\circ} \mathrm{C}$ in $5 \% \mathrm{CO}_{2}$ for 15 minutes in $0.05 \%$ collagenase type-II (PAA Laboratories, Pasching, Austria). Collagenase activity was halted using $9 \mathrm{~mL}$ of HBSS. The content was then transferred to a 15$\mathrm{mL}$ centrifuge tube, mixed by tilting the tube twice, and allowed the cells to settle down for 5 minutes. The supernatant was discarded and treated with $0.1 \%$ trypsin-ethylenediaminetetraacetic acid (Gibco-Invitrogen) for $5 \mathrm{~min}$ utes at $37^{\circ} \mathrm{C}$ to obtain tissue fragments. Then, the content was triturated in diflufenican (DFF) 10 (DMEM+F12+10\% fetal bovine serum [FBS]) to stop the trypsin action and centrifuged at 1,200 rpm for 5 minutes. The supernatant was discarded, and the cell pellet was resuspended in 1 $\mathrm{mL}$ of DFF10; $10 \mu \mathrm{L}$ of the cell suspension was mixed with $10 \mu \mathrm{L}$ of $0.4 \%$ trypan blue stain (Gibco-Invitrogen). The cells were counted using a hemocytometer under phasecontrast microscope for cell viability and total number of cells. Cells were plated approximately at a concentration of $8,000 / \mathrm{cm}^{2}$ onto polystyrene culture dishes (Greiner Bio-One, Kremsmunster, Austria) that were coated with poly-L-lysine hydrobromide $\left(2 \mu \mathrm{g} / \mathrm{cm}^{2} 0.1 \mathrm{mg} / \mathrm{mL} 30\right.$ to $70 \mathrm{kDa}$; Sigma Chemical Co., St. Louis, MO, USA). The culture dishes with these cells in the culture media (DMEM/F12 1:1 [Gibco-Invitrogen], 10\% FBS [GibcoInvitrogen], 2 mM L-glutamine [Gibco-Invitrogen]; 100 $\mathrm{U} / \mathrm{mL}$ penicillin, $100 \mu \mathrm{g} / \mathrm{mL}$ streptomycin, and $25 \mathrm{ng} / \mathrm{mL}$ of amphotericin-B) were incubated at $37^{\circ} \mathrm{C}$ in $5 \%$ carbon dioxide. The cells were fed every alternate day by replacing half of the complete culture medium. Once the cells attained $80 \%$ to $90 \%$ confluency, they were passaged.

\section{Characterization of olfactory ensheathing cell/olfac- tory nerve fibroblast using immunohistochemistry}

Cells were cultured on $12-\mathrm{mm}$ round coverslips at a cell density of 8,000 cells $/ \mathrm{cm}^{2}$. The cells were fixed in $4 \%$ paraformaldehyde (Qualigens, India) for 15 minutes at room temperature. Thereafter, the cells were washed thrice with phosphate-buffered saline (PBS, Gibco-Invitrogen). Blocking and permeabilization was performed with $2 \%$ goat serum $/ 2 \%$ bovine serum albumin (BSA; Jackson Immunoresearch Laboratories Inc., West Grove, PA, USA) with $0.1 \%$ Triton X-100/PBS (Sisco Research Laboratories Pvt. Ltd., Mumbai, India). The cells were incubated with primary antibodies, p75NTR-fluorescein isothiocyanate (FITC, 1:200; Millipore, Bedford, MA, USA), mouse antifibronectin (1:100; Santa Cruz Biotech, Santa Cruz, CA, USA), mouse monoclonal immunoglobulin G (IgG) 1 anti-S100 $\beta$ subunit (1:100, Sigma Chemical Co.), mouse monoclonal IgG1 anti-glial fibrillary acid protein (GFAP) Alexa Fluor 488 conjugated (1:50; eBioscience, San Diego, CA, USA), mouse IgG anti-GalC (1:100, Millipore) overnight at $4^{\circ} \mathrm{C}$. The cells were washed with PBS and incubated with secondary antibodies, goat anti-mouse IgG1phycoerythrin (PE) conjugated (Southern Biotechnology Associates, Birmingham, AL, USA) and goat anti-mouse IgG (H+L) Alexa Fluor 594 (1:100) for 2 hours at room temperature. They were then washed and mounted with DAPI (Vectashield mounting medium with DAPI; Vector Laboratories Inc., Burlingame, CA, USA). Coverslips were immediately transferred to glass slides and examined under a fluorescent microscope with an appropriate filter for the dye conjugated to the primary/secondary antibodies.

\section{Characterization of olfactory ensheathing cell/olfac- tory nerve fibroblast using flow cytometry}

The cultured cells were trypsinized and washed with PBS. Cell suspensions containing $2-5 \times 10^{5}$ cells were stained with a primary antibody with or without fluorescence if it was an extracellular marker. For intra-cellular protein markers, cells were fixed with $4 \%$ paraformaldehyde for 15 minutes at room temperature. This was washed with PBS following blocking and permeabilization with $2 \%$ goat serum $/ 2 \%$ BSA with $0.1 \%$ Triton X-100\PBS. We used $5-10 \mu \mathrm{L}$ of primary antibody, as mentioned above, and incubated for 20 minutes on ice. Excess unbound antibodies were washed with PBS and removed; 5-10 $\mu \mathrm{L}$ of appropriate secondary antibody was used; these were fluorescent tagged and incubated for 20 minutes. Finally, the cells were washed with PBS to remove the unbound secondary antibodies. The cell suspension was aspirated and analyzed using flow cytometry. 


\section{Green fluorescent protein labeling of cells}

Using a complete medium, $0.5 \times 10^{5} / \mathrm{mL}$ of cells were grown overnight. When the culture was $50 \%-75 \%$ confluent, $50 \mu \mathrm{L}$ of $1 \times 10^{7}$ IFU (infection function unit) $/ \mathrm{mL}$ of pre-made lentiviral particles for fluorescent proteins (catalog number, LVP001; GenTarget Inc., San Diego, CA, USA) were added. After 72 hours of transduction, the transduction rate was checked under a fluorescent microscope. The labeled cells were used for transplantation.

\section{Cell viability}

Freshly cultured second passage cells were harvested and kept on ice for 2 hours. These cells were stained for propidium iodide (PI; BD Bioscience, San Diego, CA, USA), a red fluorescent DNA counterstain. Both unstained and stained cells were quantified using flow cytometry. Dead cells were stained with PI, and live cells were not stained. Freshly harvested cells without PI were used as controls.

\section{Animal experiments}

\section{1) Experiment design}

Thirty female rats were divided into five groups ( $n=6$ rats randomly allocated to each group). OECs were transplanted at different dosages, such as $2 \times 10^{5}, 5 \times 10^{5}, 10 \times 10^{5}$, and $>10 \times 10^{5}$, in each group; in the SCI group, only DMEM was injected without OECs.

\section{2) Laminectomy and spinal cord injury}

Female Albino Wistar rats, 100-250 g in body weight, were anesthetized with ketamine $(90 \mathrm{mg} / \mathrm{kg}$; Neon Laboratories Ltd., Mumbai, India) and xylazine (10 mg/kg; Indian Immunologicals Ltd., Hyderabad, India) that was administered intraperitoneally. Ophthalmic ointment (neomycin; Glaxosmithkline Pharmaceuticals Ltd., Bangalore, India) was applied to the eyes to prevent drying during the operation. The fur was shaved on the middorsal region and cleaned with povidone-iodine solution (7.5\% w/v; Wockhardt Ltd., Mumbai, India) and finally with surgical spirit (Kakatiya Pharma, Hyderabad, India). Tega-derm (3M Tegaderm film; 3M Health Care, St. Paul, MN, USA) was applied over it to prevent fur contamination during surgery. A $2-\mathrm{cm}$ incision was made over the lower thoracic area; the muscle and the connective tissue were bluntly dissected to expose the T6-T9 vertebrae. A
T10 spinal level laminectomy was completed using a microsurgery bone rongeur to expose the spinal cord. The vertebral bodies were stabilized using customized vertebral clamps, and the impact rod was positioned over the spinal cord. Drop-weight injury was performed, wherein a 10 - $\mathrm{g}$ rod was made to fall on the spinal cord from a height of $25 \mathrm{~cm}$. An absorbable suture (vicryl; Johnson-Johnson Ltd., New Brunswick, NJ, USA) was used to ligate the incised muscle and skin. Animals had free access to food and water throughout the study. Bladder and bowel expressed as postoperative care.

In a standardization study, mild contusion injury did not show complete paralysis of the hind limb in a rat model. Therefore, we increased the height to $25 \mathrm{~cm}$, i.e., the 10-g rod was made to fall from a height of $25 \mathrm{~cm}$ to create the drop-weight injury. Thus, complete injury was created, and no spontaneous hind limb motor recovery was noticed even after several months. Paraplegic, motorevoked potential studies were performed for the continuation of spinal tracts from the motor cortex to the hind limb muscles. On stimulation of the motor cortex, there was no muscle EMG amplitude. This shows that the tracts are cut (discontinuous), and there is no impulse propagation. This analysis was performed to verify and validate the model used in our study.

\section{3) Postoperative care}

Following the surgery, the rats were placed in a cage and monitored until they recovered from anesthesia. The rats were monitored twice a day throughout the postinjury survival period for general health and mobility within the cage. The bladder was manually expressed twice daily. Ringer lactate $(5 \mathrm{~mL} / 100 \mathrm{~g}$; Claris Lifescience Ltd., Ahmedabad, India) was administered subcutaneously twice daily after each bladder expression on the first postoperative 7 days. Meloxicam (1 mg/kg; Intas Pharmaceuticals Ltd., Ahmedabad, India), as an analgesic, and enrofloxacin (2.5 mg/kg, Intas Pharmaceuticals Ltd.), as an antibiotic, were administered for the first postoperative 7 days. The animals were monitored for urinary tract infections (UTI) and general symptoms, such as feeling unwell, listlessness, turbid urine, febrile, tachypnoea, and blood-stained urine, for the entire experimental period. If UTI was indicated, they were treated with antibiotics (enrofloxacin $2.5 \mathrm{mg} / \mathrm{kg}$ ). Inspection for skin ulcers was conducted daily. The bedding was changed every alternate day. 
4) Cell transplantation

Allogenic cell transplantation was performed on the 9th day following the drop-weight injury. Behavioral assessment (BBB score) was conducted prior to the cell transplantation, as described below. Rats were re-anesthetized (intraperitoneal ketamine/xylazine: $90: 10 \mathrm{mg} / \mathrm{kg}$ ), and the original incision was re-opened to the T6-T9 vertebrae. Under a surgical microscope, the wound was explored, and the injured spinal cord segment as well as the region a few millimeter above and below the normal spinal cord was exposed. On the day of the transplantation, the OEC/ ONF were harvested by trypsinization; thereafter, the enzymatic activity was stopped by adding DMEM with fetal bovine serum. The cells were counted and pelleted, transferred into a $25-\mu \mathrm{L}$ Hamilton syringe (approximately 100,000 cells $/ \mu \mathrm{L}$ ). A bevel tip needle of $0.035 \mathrm{~mm}$ diameter was attached to a $25-\mu \mathrm{L}$ Hamilton syringe mounted on an injection device with a three-dimensional stabilizer. All the injections were prepared with the help of a sterile Hamilton syringe. Cell suspensions in the doses: $2 \times 10^{5}$, $5 \times 10^{5}, 10 \times 10^{5}$, and $>10 \times 10^{5}$ were injected at multiple sites $(2 \mu \mathrm{L} /$ site $)$ in and around the injury epicenter of the injured spinal cord. Following cell transplantation, the surgical wound was closed, and routine postoperative care was given.

5) Behavioral assessment using Basso, Beattie, Bresnahan score

The BBB scale is an operationally defined 21-point scale designed to assess hind limb locomotor recovery after injury to the spinal cord in rats [15]. This locomotor scale categories the combinations of rat hind limb joint movements, trunk position and stability, stepping, coordination, paw placement, toe clearance, and tail position, representing sequential recovery stages that rats experience after SCI. The motor assessments were performed upto 8-10 weeks after injury/transplantation. Open-field observations were performed for the rats. All the rats received bladder expression before open-field testing to eliminate behaviors due to bladder fullness. Rats were allowed to walk in the open field $(45 \mathrm{~cm} \times 60 \mathrm{~cm}$ rectangular tray) and videographed for assessment. All the rats were assessed for BBB before transplantation, i.e., on the 9th day after SCI and every week after the transplantation for up to $8-10$ weeks. The BBB score was calculated with the aid of a BBB scale.
6) Motor-evoked potential studies

After 8-10 weeks post-injury/transplant, all the rats were anesthetized with ketamine $(90 \mathrm{mg} / \mathrm{kg}$ ) and xylazine (10 $\mathrm{mg} / \mathrm{kg}$ ) with an intraperitoneal injection. EMG stimulator device and recording software (CMCdaq) were designed by the Department of Bioengineering, Christian Medical College, Vellore [16]. Scalp fur was removed, and a superficial bipolar electrode was placed on the scalp for transcranial electrical stimulation of the motor cortex; their EMG signals were recorded from the hind limb gastrosoleus muscle by placing a needle electrode; $1.5-\mathrm{V}$ alkaline batteries were used in the pulse stimulator. The signals were amplified, filtered (10-5,000 Hz), and sampled at $2 \mathrm{kHz}: 0.050 \mathrm{sec} / \mathrm{sweep}$ of the EMG channel. The output of the data acquisition was $0.050 \mathrm{sec} / \mathrm{screen}, 1.500$ $\mathrm{mV} /$ div. The maximum peak attained in the wave pattern was considered for amplitude, and the mean amplitude value of three random recorded waves were calculated for each rat. Single channel recorded EMG signals were analyzed for the amplitude in $\mathrm{mV}$; data are represented as mean \pm standard deviation (SD) values in all the groups and compared using $p$-values. This parameter was used to evaluate the functional integrity of the spinal cord.

\section{7) Histology}

Two weeks after green fluorescent protein (GFP)-labeled OEC/ONF transplantation, the rats were deeply anesthetized with ketamine/xylazine and transcardially perfused with $4 \%$ paraformaldehyde solution. Thereafter, $1-\mathrm{cm}$ length of the spinal cord centered on the injury epicenter was removed and post-fixed in 30\% sucrose/phosphatebuffered saline overnight at $4^{\circ} \mathrm{C}$. Twenty micrometer $(\mu \mathrm{m})$-thick longitudinal serial cryo-sections were cut and mounted on poly-L-Lysine coated slides. A representative tissue section was washed with PBS and visualized under a confocal microscope.

\section{Statistical analyses}

Hind limb motor recovery BBB scores and the amplitude of motor-evoked potential were statistically analyzed using SPSS ver. 16.0 (SPSS Inc., Chicago, IL, USA), analysis of variance, and Post Hoc Tukey to compare the differences between the two groups. Paired (pre- and post-transplant BBB scores) analyses was conducted using the Wilcoxon test. Data for each group were represented as mean \pm SD. All $p$-value $<0.05$ was considered statistically significant. 

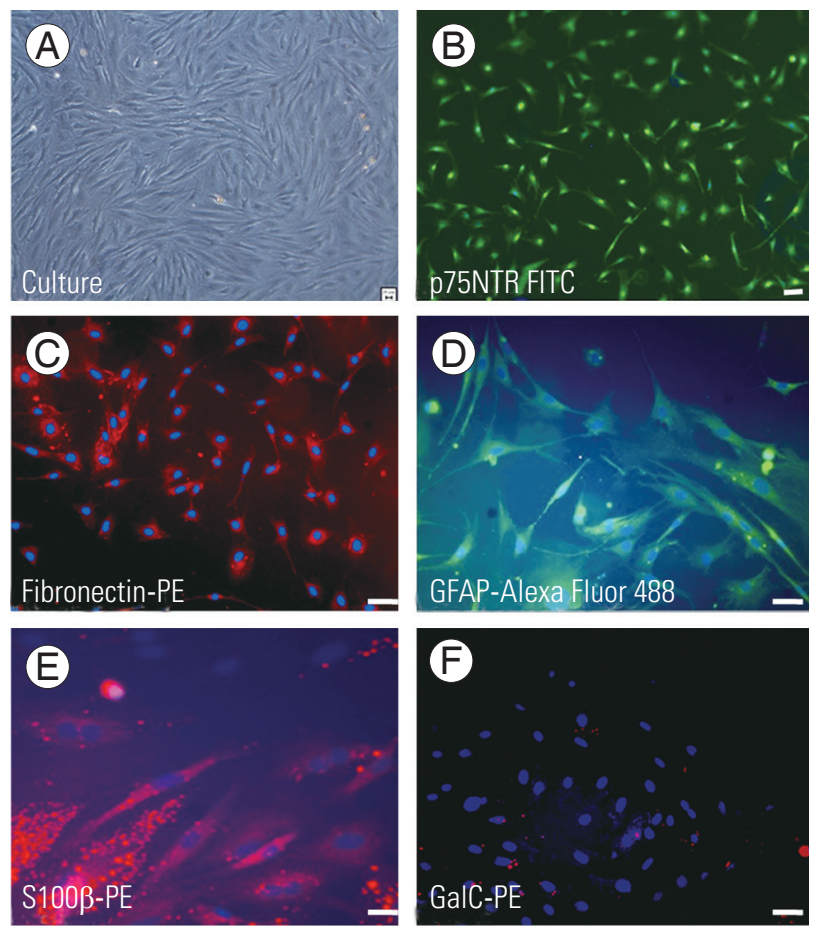

Fig. 1. Immunohistochemical characterization of OECs and ONFs. Image showing culture in phase contrast (A), OECs stained with P75NTR-fluorescein isothiocyanate (B), and ONF positively stained with fibronectin-PE (C). OEC/ONF stained with GFAP-Alexa Fluor 488 (D) and S100ß-PE (E) showing positivity for astrocyte and Schwann cell markers. (F) OEC/ONF stained with GalC-PE showing negativity for the marker. Scale bar $=20 \mu \mathrm{m}$. OEC, olfactory ensheathing cell; ONF, olfactory nerve fibroblast; $\mathrm{PE}$, phycoerythrin.
Wilcoxon test was used to compare the treatment group before the transplant (on the 9th day after SCI) and after the transplant (8th week after OEC transplantation).

\section{Results}

\section{Characterization of the cultured cells}

Rat OEC and ONF were immunohistochemically characterized using p75NTR-FITC and fibronectin-PE, respectively. Cultured OEC/ONF cells expressed S100 $\beta$ (Schwann cell marker) and GFAP (Astrocyte marker) and were negative for Galc, a marker for oligodendrocytes (Fig. 1). Flow cytometry analyses showed p75NTR of $41 \%$ positive for OEC and $35 \%$ positive for anti-fibronectin, a marker for ONF. In addition, OEC/ONFs were found to express S100 $\beta$ and GFAP of approximately $75 \%$ and $83 \%$, respectively (Fig. 2).

\section{Cell viability}

The viability of cells was assessed using PI, a red fluorescent DNA counterstain, that showed that only $2 \%$ of the cells were dead; the remaining $98 \%$ cells were viable for transplantation (Fig. 3).
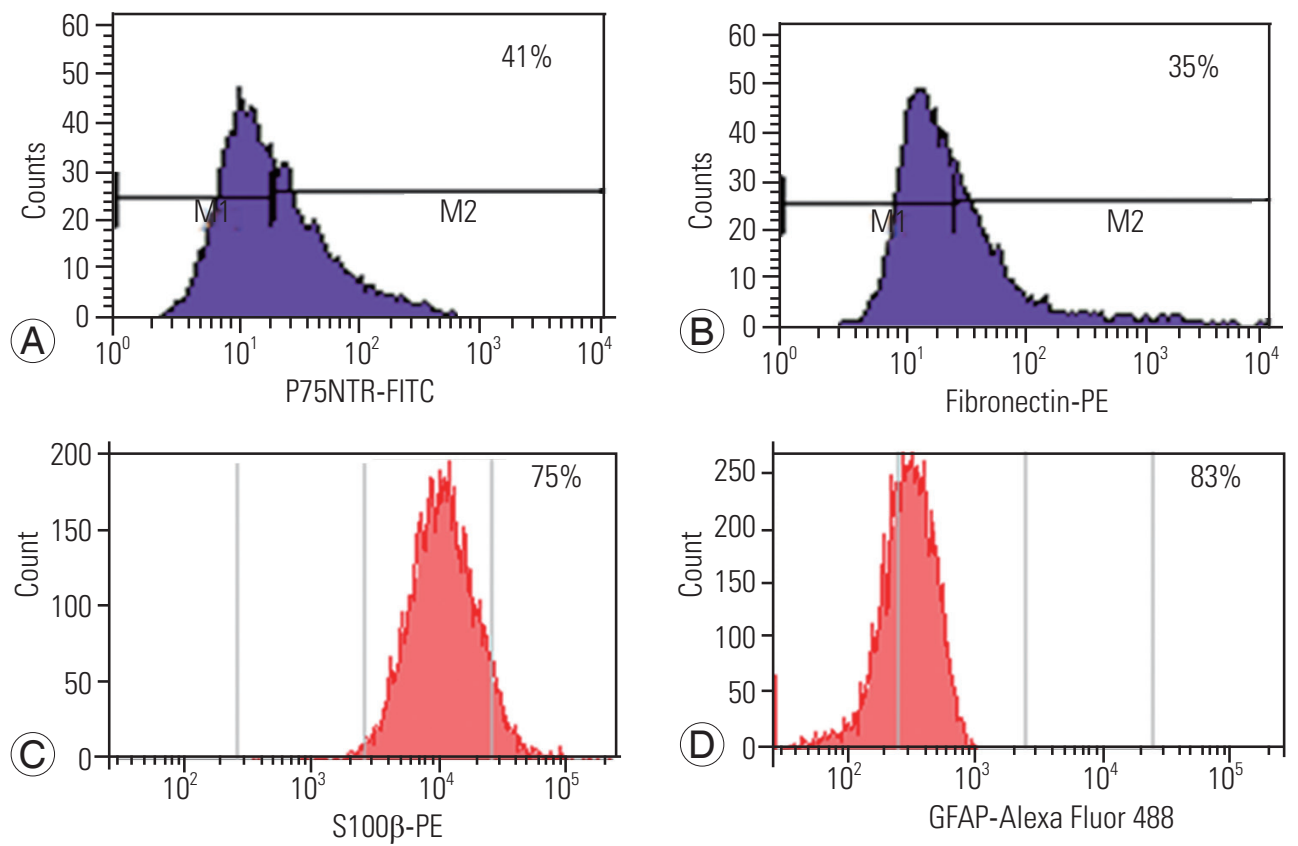

Fig. 2. Flow cytometric characterization of OEC/ONF. Analysis of P75NTR-FITC showing 41\% positivity for OEC (A), and fibronectinPE showing 35\% positivity for ONF (B). Cells were 75\% positive for S100ß-PE (C) and 83\% positive for GFAP-Alexa Fluor 488 (D). $\mathrm{OEC}$, olfactory ensheathing cell; ONF, olfactory nerve fibroblast; PE, phycoerythrin; FITC, fluorescein isothiocyanate. 

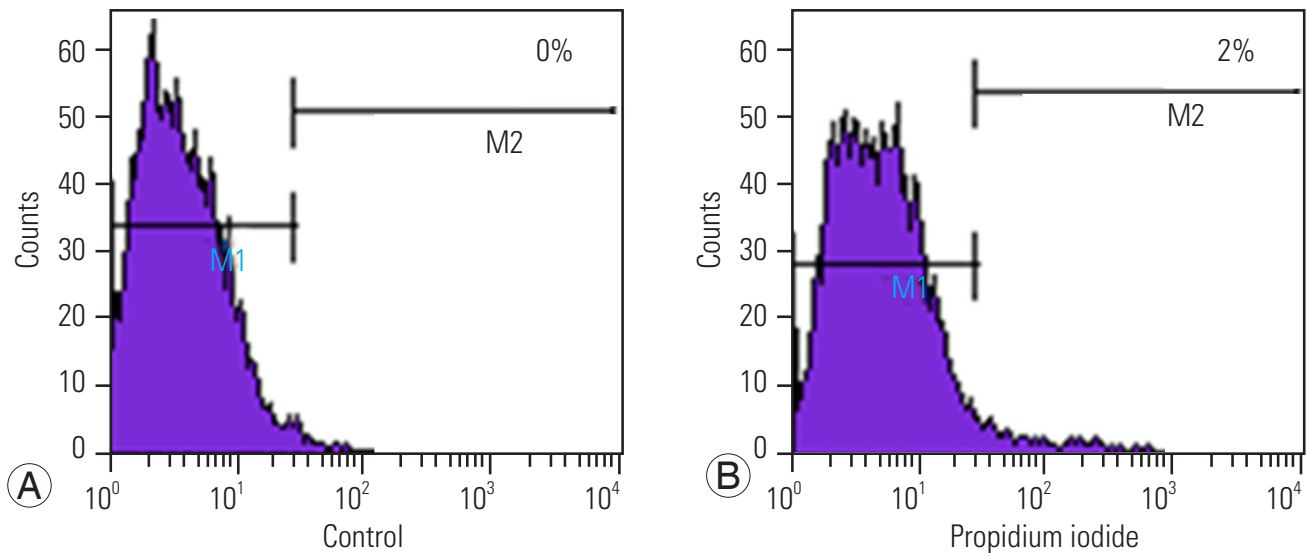

Fig. 3. Quantification of viable olfactory ensheathing cells after culture harvest. (A, B) Unstained cells used as controls; propidium iodide staining showing $2 \%$ positivity, indicating $98 \%$ viable cells.

\begin{tabular}{|c|c|c|c|c|c|c|c|c|c|}
\hline \multirow[b]{2}{*}{ Groups } & After SCl & \multicolumn{8}{|c|}{ BBB transplant } \\
\hline & $\begin{array}{l}\text { Before } \\
\text { transplant/9th } \\
\text { day after SCl }\end{array}$ & $\begin{array}{l}\text { 1st } \\
\text { wk }\end{array}$ & $\begin{array}{l}\text { 2nd } \\
\text { wk }\end{array}$ & $\begin{array}{l}3 \text { rd } \\
\text { wk }\end{array}$ & $\begin{array}{l}4 \text { th } \\
\text { wk }\end{array}$ & $\begin{array}{l}\text { 5th } \\
\text { wk }\end{array}$ & $\begin{array}{l}\text { 6th } \\
\text { wk }\end{array}$ & $\begin{array}{l}\text { 7th } \\
\text { wk }\end{array}$ & $\begin{array}{l}\text { 8th } \\
\text { wk }\end{array}$ \\
\hline OEC2L (n=6) & 0 & 0.6 & 1 & 2.1 & 2.5 & 3 & 3 & 3 & 3 \\
\hline OEC5L $(n=6)$ & 0.1 & 2.1 & 4.8 & 5.6 & 6.1 & 6.5 & 6.6 & 7.1 & 7.1 \\
\hline OEC10L $(n=6)$ & 0.1 & 1.8 & 2.6 & 3 & 4.1 & 4.8 & 4.8 & 4.8 & 5.3 \\
\hline OEC $>10 \mathrm{~L}(\mathrm{n}=6)$ & 0 & 1.8 & 3.3 & 4.5 & 4.8 & 5.3 & 5.8 & 5.8 & 5.8 \\
\hline $\mathrm{SCl}(\mathrm{n}=6)$ & 0.1 & 0.1 & 0.1 & 0.1 & 0.1 & 0.1 & 0.1 & 0.1 & 0.1 \\
\hline
\end{tabular}
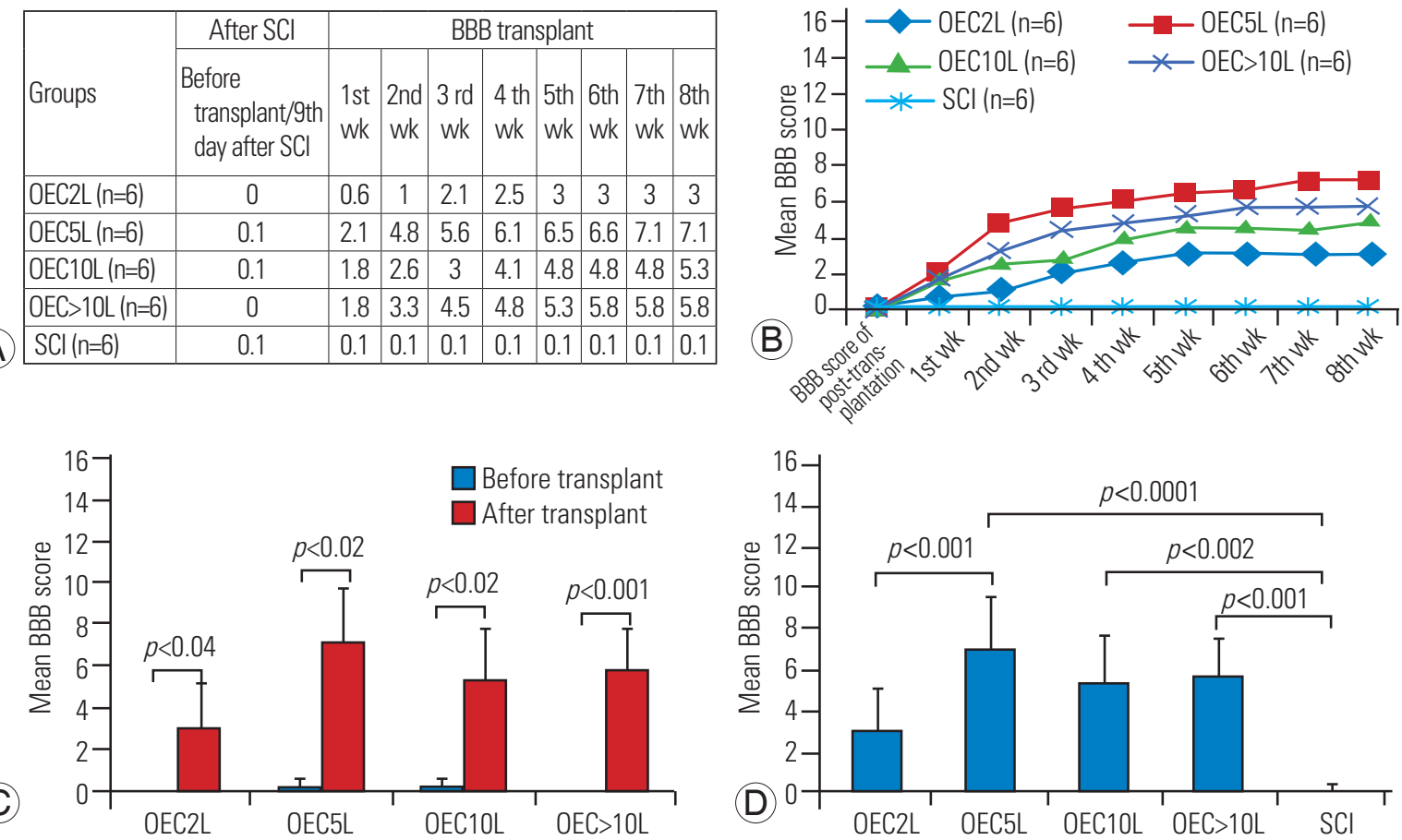

Fig. 4. Mean BBB score for hind limb motor recovery. All the transplanted groups showed improved BBB scores, except the SCI group, as shown in (A, B). Within-group comparisons before and after transplantation showed significant functional recovery after transplantation $(p<0.05)$ (C). A comparison among the groups showed that 5,10 , and $>10 \times 10^{5}$ cell groups demonstrated significant improvements in motor function $(p<0.05)$ compared with the SCl group. While all the transplanted groups showed functional recovery, only the 2 and $5 \times 10^{5}$ cell groups showed significant differences (D). BBB, Basso, Beattie, Bresnahan scale; SCl, spinal cord injury; OEC, olfactory ensheathing cell.

\section{Basso, Beattie, Bresnahan score}

The different dosages of OEC transplantation showed sequential recovery from the 1st week post-transplant and were followed up till the 8 th week. Five lakh treated rats showed the highest motor score of 7.1 among the treated groups. The SCI group did not show progress in hind limb motor recovery. The 2-lakh group showed minimum recovery as compared to the other transplanted groups (Fig. $4 \mathrm{~A}, \mathrm{~B})$.

Following SCI, the BBB score was assessed on the 9th day, that is, before the transplant when all the groups 
showed a mean score of zero. After transplantation, with different dosages of cells, hind limb motor recovery improved by the end of the 8th week. The mean BBB score with $2 \times 10^{5}$ cells was $(3.0 \pm 2.19)$, that with $5 \times 10^{5}$ cells was $7.1 \pm 2.56$, that with $10 \times 10^{5}$ cells was $5.3 \pm 2.50$, and that with $>10$ lakh cells was $5.8 \pm 1.83$. All the rats that underwent OEC transplantation after SCI with different dosages showed significant motor recovery, as measured with the BBB score $(p<0.05)$ (Fig. 4C).

As shown in Fig. 4D, the effect of different dosages of cell transplantation was compared. The 5-lakh cell group showed a significant improvement in the motor score as compared to the 2-lakh cell group $(p<0.01)$. Comparing the motor outcome of low dose of $2 \times 10^{5}$ cells with high dose of $\geq 10$ lakh cells showed no significant difference $(p>0.05)$; however, a functional difference was elicited. Similarly, in the 5-lakh and 10-lakh cell groups, there was no statistically significant difference in the BBB score $(p<0.545)$.

The motor score of the transplantation group was compared with that of the SCI group $(0.1 \pm 0.40)$. The treated groups (5 [7.1 \pm 2.56$], 10[5.3 \pm 2.50]$, and $>10 \times 105[5.8 \pm 1.83])$ showed significantly higher motor score $(p<0.05)$ than the SCI group; however, no such difference was observed with the $2 \times 10^{5}$ cells group $(p<0.153)$ (Fig. 4D).

\section{Motor-evoked potential study}

Transcranial stimulation of the motor cortex was performed, and EMG was recorded from the gastrosoleus for analysis in all the groups. Transplantation of $2 \times 10^{5}$ cells did not show any significant changes in the EMG amplitude compared to all other groups, including the SCI group. Compared to the SCI group, the 5 and $10 \times 10^{5}$ cell groups showed significant improvement in the EMG amplitude $(p<0.05)$. However, the $2(0.69 \pm 0.33)$ and $>10 \times 10^{5}$ cell groups $(0.56 \pm 0.04)$ showed no significant difference in the amplitude compared with the SCI group $(0.30 \pm 0.21)$.

There was no difference in the amplitude of the 5-lakh group (1.20 \pm 0.41$)$ and the 10-lakh group (1.21 \pm 0.48$)$ $(p<1.000)$. The amplitude of the 5-lakh group showed an increase $(1.20 \pm 0.41)$ when compared to that of the $>10$-lakh group (0.5 \pm 0.04$)$; the difference was significant $(p<0.024)$. The 10-lakh group $(1.21 \pm 0.48)$ analyzed had a significantly higher amplitude than the $>10$-lakh group $(0.56 \pm 0.04)(p<0.023)$. Both the 5-lakh $(1.20 \pm 0.41)$ and 10-lakh (1.21 \pm 0.48$)$ groups showed higher amplitudes
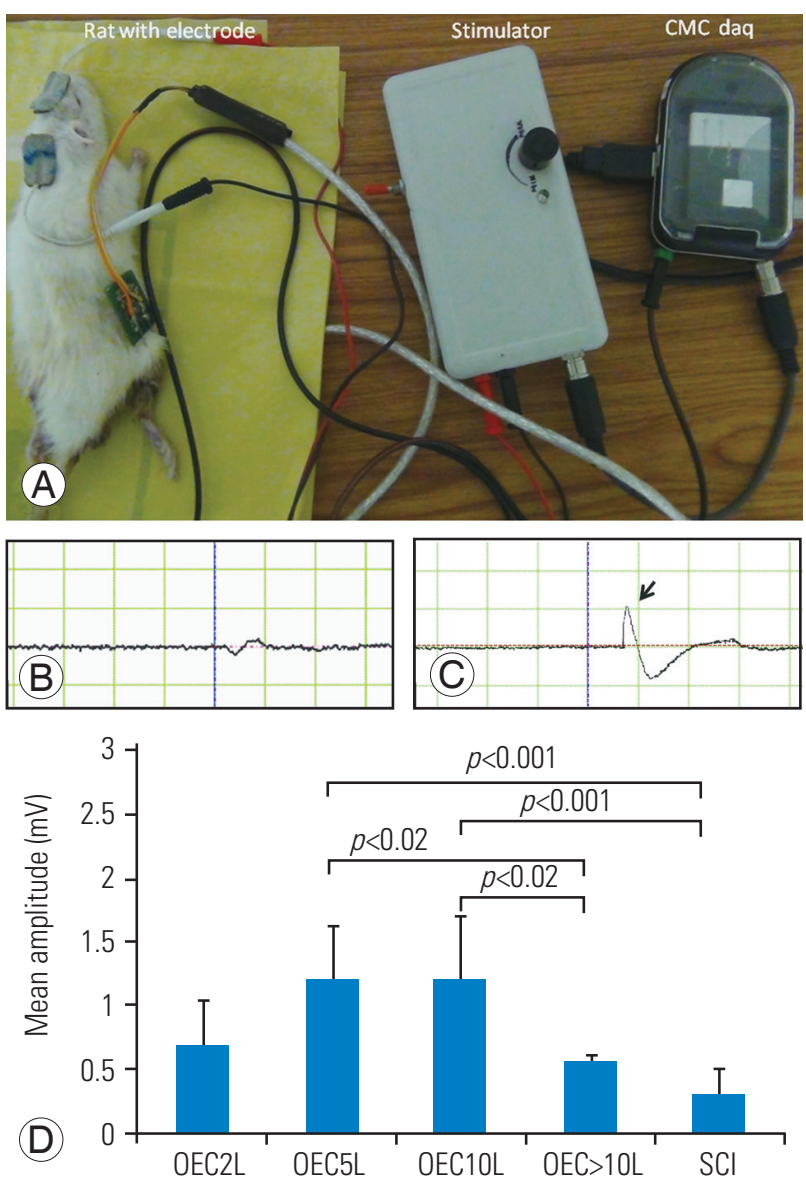

Fig. 5. Transcranial electrical stimulation and hind limb motor-evoked potential studies. Motor cortex stimulation and their responses were recorded in hind limb muscle 8 weeks after the transplantation in anesthetized rats (A). On stimulation, there was no amplitude seen in the control rats $(\mathbf{B})$; however, the representative electromyographic amplitude in the transplanted rats $\left(5 \times 10^{5}\right)$ is shown by the arrow (C). Amplitude varied based on regeneration in the different groups. Statistical analyses showed significant differences $(p<0.05)$ in the 5 and $10 \times 10^{5}$ cell groups compared with the control group; however, the other groups showed no significant differences in the mean amplitude. The 5 and $10 \times 10^{5}$ cell groups elicited a significant response compared with the $>10 \times 10^{5}$ cell group (D).

(Fig. 5). However, all the treated groups exhibited different amounts of regeneration, except the SCI group, according to EMG study.

\section{Histology}

GFP-labeled OECs/ONFs were observed for survival around the injury epicenter of the cord (Fig. 6). These surviving cells probably favored the recovery of hind limb motor function in spinal cord injured rats in the transplanted groups. 

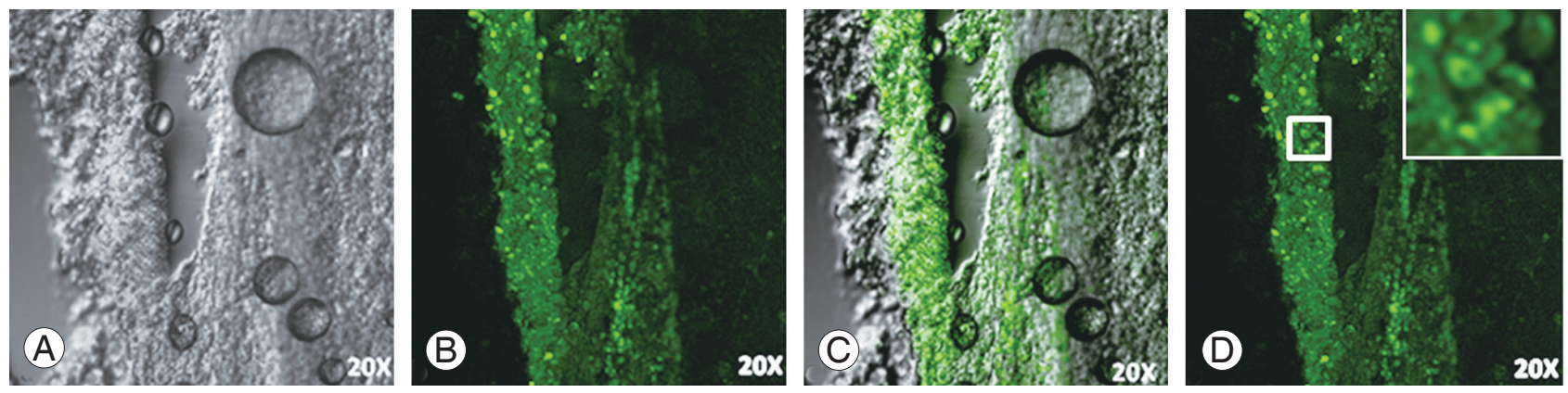

Fig. 6. (A-D) Histology of the rat spinal cord. Green fluorescent protein-labeled rat OECs survived in injured rat spinal cord until 2 weeks post transplantation. (A) Phase contrast, (B) GFP cells around the injury epicenter, (C) Merged image of A and B showing GFP-labeled OECs in the spinal cord transection (20x magnification). OEC, olfactory ensheathing cell; GFP, green fluorescent protein.

\section{Discussion}

This study aimed to observe the role of OEC transplantation for neural repair following SCI in rat models. Different dosages of cells were used for transplantation, and the outcome was monitored over time. Extensive research using cell therapy with a wide range of cells is being conducted worldwide. They include pre-oligodendrocytes, macrophages, mesenchymal stem cells, Schwann cells, and OECs. OECs have attracted attention because they are easily accessible and involve a less invasive procedure for olfactory tissue collection; additionally, they are derived form neurogenic tissue (cells isolated from ectoderm and transplanted to the ectoderm-derived spinal cord) and possess regenerative potential. In future clinical applications, olfactory mucosa can be isolated from patients with SCI and transplanted to the injured spinal cord. These are autologous; therefore, there are no ethical issues or graft versus host disease. However, the collection of olfactory mucosa from the rat and transplantation to the injured spinal cord of the same rat is technically challenging. Therefore, allogenic transplantation was done. OECs have characteristics of the cells of the CNS and the peripheral nervous system.

In a comparative study of OEC and Schwann cell transplantation for contused SCI that assessed the outcomes for axonal growth, myelination, and functional recovery of the hind limb, Schwann cells exhibited greater reparative effect than OECs [17]. However, the Schwann cells did not mingle with the astrocyte-rich environment and induced hypertrophy of the scar by upregulation of chondroitin sulfate proteoglycans $[18,19]$. Thus, the Schwann cells were considered unsuitable for transplantation in spinal cord regeneration [20].

In our study, we found that cultured cells expressed $41 \%$ of p75NTR, a marker for OEC, and 35\% of anti-fibronectin, a marker for ONF. These cells also expressed S100 $\beta$ and GFAP, $75 \%$ and $83 \%$, respectively. These markers confirm CNS glial astrocyte characteristics as well as peripheral nervous system glial Schwann cell characteristics for the cultured OEC. Other studies suggest that OECs are $\mathrm{p} 75^{+}$and ONFs are fibronectin positive. OEC are positive for p75 marker and weakly positive for GFAP. The olfactory mucosa culture have a proportion of 50:50 OEC/ ONF ratio, than olfactory bulb [21].

For successful repair, OEC transplantation in combination with ONF has shown better outcomes than OEC alone. Normally, the olfactory neurons are guided by OEC in relationship with ONF in the olfactory system. Similarly, OEC with ONF are necessary for transplant-mediated repair in SCI [22]. Studies have shown that OEC enwrap the host nerve fibers initially and form myelin sheaths after 3-4 weeks. This myelin sheath acts as peripheral nerve myelin, similar to Schwann cells, but unlike the myelin in the CNS produced by oligodendrocytes [23,24].

The antigenic profile of olfactory mucosa OEC and olfactory bulb OEC were similar in culture; however, they exhibited different functional behavior after transplantation. Rostrocaudal transplant showed axonal sprouting across the lesion site, while direct transplant was effective in enhancing the axonal growth toward the lesion boundaries; few axons were able to penetrate the lesion. This effect may be attributable to both trophic/tropic actions of OEC and changes in the permissive environment of the glial scar/lesion site that ultimately resulted in decreased GFAP reactivity and cavity formation [25]. 
In our study, we focused on cell viability, dose, therapeutic window period, and route of administration. Total $98 \%$ of the cells were viable, and only $2 \%$ were non-viable, confirming that live cells were transplanted. Initially after the trauma, the inflammatory response shoots up and slowly declines by the 9th day. Therefore, transplantation was performed on the 9th day after the SCI to prevent cell loss due to inflammatory responses. The therapeutic window period for transplantation is also a key factor, as evidenced by cell engraftment and improved functional outcome in cases where transplantation is performed within 14 days of injury [26,27]. To elucidate the doseresponse relationship, we tried different dosages of $2 \times 10^{5}$ (2 lakh), $5 \times 10^{5}$ (5 lakh), $10 \times 10^{5}$ (10 lakh), and $>10 \times 10^{5}$ (>10 lakh) for transplantation. In this study, 5-10 lakh cell dosage had the best therapeutic effect. Poor response to low dosage could be due to the inadequate number of cells. In very high dosage $\left(10 \times 10^{5}\right.$ [10 lakh] and $>10 \times 10^{5}$ [>10 lakh]) of cell transplantation, reduced motor recovery may be due to the saturation effect or the inability to accommodate in the cord, resulting in unsatisfactory outcomes. We have transplanted cells in and around the injured spinal cord as a therapeutic strategy rather than as an infusion or injection in the lumbar subarachnoid space because such routes depend on homing mechanisms.

The BBB score improved in all the transplanted groups, expect the SCI group. In addition to higher BBB scores, motor-evoked potential showed increased amplitudes in the treated groups. In vivo functional recovery after OEC the transplantation study reported axonal regeneration and electrophysiological evidence of the recovery of motor-evoked potentials in complete transection lesion [28]. Another study indicated functional improvements due to trophic support mechanism of the grafted OEC [29]. OECs are able to preserve the function of circuitry with sensorimotor cortex potentials and evoked cord dorsum potentials in the region of lesion dorsal column after transplantation [13]. In another study, Liu et al. [30] showed significant improvement in the behavior, motorevoked potential, and somatosensory potentials in rats after OEC transplantation. Among the different dosages of OEC transplantation, $5 \times 10^{5}$ cells were observed to result in best motor outcomes in BBB and motor-evoked potentials. Even though the quantification of regeneration was not performed histologically, GFP-labeled OECs were observed to survive in the injured cord that could be responsible for transplant-mediated repair. There was a limitation regarding histological evaluation before and after transplantation in this study.

\section{Conclusions}

This dose-response relationship study suggests that the transplantation of five lakh OEC/ONF has the best therapeutic beneficial effect in rat models of SCI. This may have implications in the dose calculation for future human cell transplantation. These cells could be candidates for autologous transplantation and are easily accessible with minimal damage, with regenerative potential for clinical application following SCI. Our results are encouraging and recommend further research. Studies involving larger samples sizes and higher mammals study are recommended.

\section{Conflict of Interest}

No potential conflict of interest relevant to this article was reported.

\section{Acknowledgments}

This study was supported by Department of Biotechnology, Ministry of Science \& Technology, Government of India (fund no., BT/PR12619/MED/31/70/2009).

\section{ORCID}

Durai Murugan Muniswami:

https://orcid.org/0000-0001-6838-9727

\section{Author Contributions}

Durai Murugan Muniswami: experiment done, statistical analysis, data interpretation and manuscript preparation; and George Tharion: fund collection, study designed, supervised and manuscript preparation.

\section{References}

1. Kwon BK, Borisoff JF, Tetzlaff W. Molecular targets for therapeutic intervention after spinal cord injury. Mol Interv 2002;2:244-58.

2. Lin XY, Lai BQ, Zeng X, et al. Cell transplantation and neuroengineering approach for spinal cord 
injury treatment: a summary of current laboratory findings and review of literature. Cell Transplant 2016;25:1425-38.

3. Tso D, McKinnon RD. Cell replacement therapy for central nervous system diseases. Neural Regen Res 2015;10:1356-8.

4. Mackay-Sim A. Neurogenesis in the adult olfactory neuroepithelium. In: Doty RL, editor. Handbook of olfaction and gustation. New York (NY): Dekker; 2003. p. 93-113.

5. Barnett SC. Olfactory ensheathing cells: unique glial cell types? J Neurotrauma 2004;21:375-82.

6. Li Y, Carlstedt T, Berthold CH, Raisman G. Interaction of transplanted olfactory-ensheathing cells and host astrocytic processes provides a bridge for axons to regenerate across the dorsal root entry zone. Exp Neurol 2004;188:300-8.

7. Boruch AV, Conners JJ, Pipitone M, et al. Neurotrophic and migratory properties of an olfactory ensheathing cell line. Glia 2001;33:225-9.

8. Woodhall E, West AK, Chuah MI. Cultured olfactory ensheathing cells express nerve growth factor, brainderived neurotrophic factor, glia cell line-derived neurotrophic factor and their receptors. Brain Res Mol Brain Res 2001;88:203-13.

9. Imaizumi T, Lankford KL, Waxman SG, Greer CA, Kocsis JD. Transplanted olfactory ensheathing cells remyelinate and enhance axonal conduction in the demyelinated dorsal columns of the rat spinal cord. J Neurosci 1998;18:6176-85.

10. Li Y, Decherchi P, Raisman G. Transplantation of olfactory ensheathing cells into spinal cord lesions restores breathing and climbing. J Neurosci 2003;23:72731.

11. Barbour HR, Plant CD, Harvey AR, Plant GW. Tissue sparing, behavioral recovery, supraspinal axonal sparing/regeneration following sub-acute glial transplantation in a model of spinal cord contusion. BMC Neurosci 2013;14:106.

12. Roet KC, Verhaagen J. Understanding the neural repair-promoting properties of olfactory ensheathing cells. Exp Neurol 2014;261:594-609.

13. Toft A, Scott DT, Barnett SC, Riddell JS. Electrophysiological evidence that olfactory cell transplants improve function after spinal cord injury. Brain 2007;130(Pt 4):970-84.

14. Ziegler MD, Hsu D, Takeoka A, et al. Further evi- dence of olfactory ensheathing glia facilitating axonal regeneration after a complete spinal cord transection. Exp Neurol 2011;229:109-19.

15. Basso DM, Beattie MS, Bresnahan JC. A sensitive and reliable locomotor rating scale for open field testing in rats. J Neurotrauma 1995;12:1-21.

16. Prabhav NR, Devasahayam SR. Effect of muscle length changes on classification of EMG for prosthesis control. Int J Biomed Eng Technol 2013;13:11732.

17. Takami T, Oudega M, Bates ML, Wood PM, Kleitman N, Bunge MB. Schwann cell but not olfactory ensheathing glia transplants improve hindlimb locomotor performance in the moderately contused adult rat thoracic spinal cord. J Neurosci 2002;22:6670-81.

18. Barnett SC, Chang L. Olfactory ensheathing cells and CNS repair: going solo or in need of a friend? Trends Neurosci 2004;27:54-60.

19. Lakatos A, Barnett SC, Franklin RJ. Olfactory ensheathing cells induce less host astrocyte response and chondroitin sulphate proteoglycan expression than Schwann cells following transplantation into adult CNS white matter. Exp Neurol 2003;184:23746.

20. Wewetzer K, Verdu E, Angelov DN, Navarro X. Olfactory ensheathing glia and Schwann cells: two of a kind? Cell Tissue Res 2002;309:337-45.

21. Jani HR, Raisman G. Ensheathing cell cultures from the olfactory bulb and mucosa. Glia 2004;47:130-7.

22. Raisman G. Olfactory ensheathing cells and repair of brain and spinal cord injuries. Cloning Stem Cells 2004;6:364-8.

23. Barnett SC, Alexander CL, Iwashita Y, et al. Identification of a human olfactory ensheathing cell that can effect transplant-mediated remyelination of demyelinated CNS axons. Brain 2000;123(Pt 8):1581-8.

24. Kato T, Honmou O, Uede T, Hashi K, Kocsis JD. Transplantation of human olfactory ensheathing cells elicits remyelination of demyelinated rat spinal cord. Glia 2000;30:209-18.

25. Richter MW, Fletcher PA, Liu J, Tetzlaff W, Roskams AJ. Lamina propria and olfactory bulb ensheathing cells exhibit differential integration and migration and promote differential axon sprouting in the lesioned spinal cord. J Neurosci 2005;25:10700-11.

26. Okano H. Stem cell biology of the central nervous system. J Neurosci Res 2002;69:698-707. 
27. Torres-Espin A, Hernandez J, Navarro X. Gene expression changes in the injured spinal cord following transplantation of mesenchymal stem cells or olfactory ensheathing cells. PLoS One 2013;8:e76141.

28. Ziegler MD, Hsu D, Takeoka A, et al. Further evidence of olfactory ensheathing glia facilitating axonal regeneration after a complete spinal cord transection. Exp Neurol 2011;229:109-19.

29. Lu P, Yang H, Culbertson M, Graham L, Roskams
AJ, Tuszynski MH. Olfactory ensheathing cells do not exhibit unique migratory or axonal growth-promoting properties after spinal cord injury. J Neurosci 2006;26:11120-30.

30. Liu KJ, Xu J, Yang CY, et al. Analysis of olfactory ensheathing glia transplantation-induced repair of spinal cord injury by electrophysiological, behavioral, and histochemical methods in rats. J Mol Neurosci 2010;41:25-9. 